
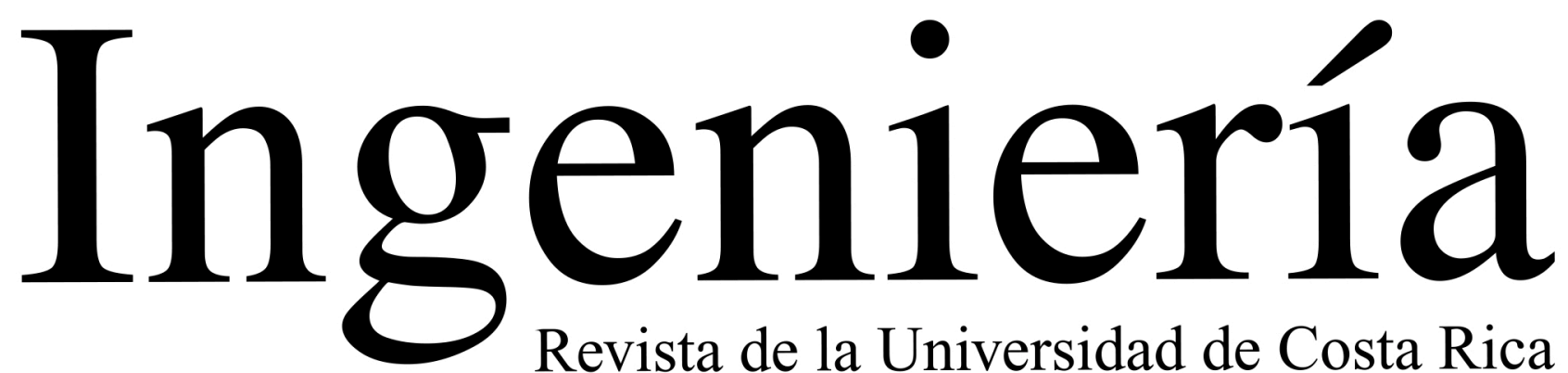

Revista de la Universidad de Costa Rica JULIO/DICIEMBRE 2021 - VOLUMEN 31 (2)

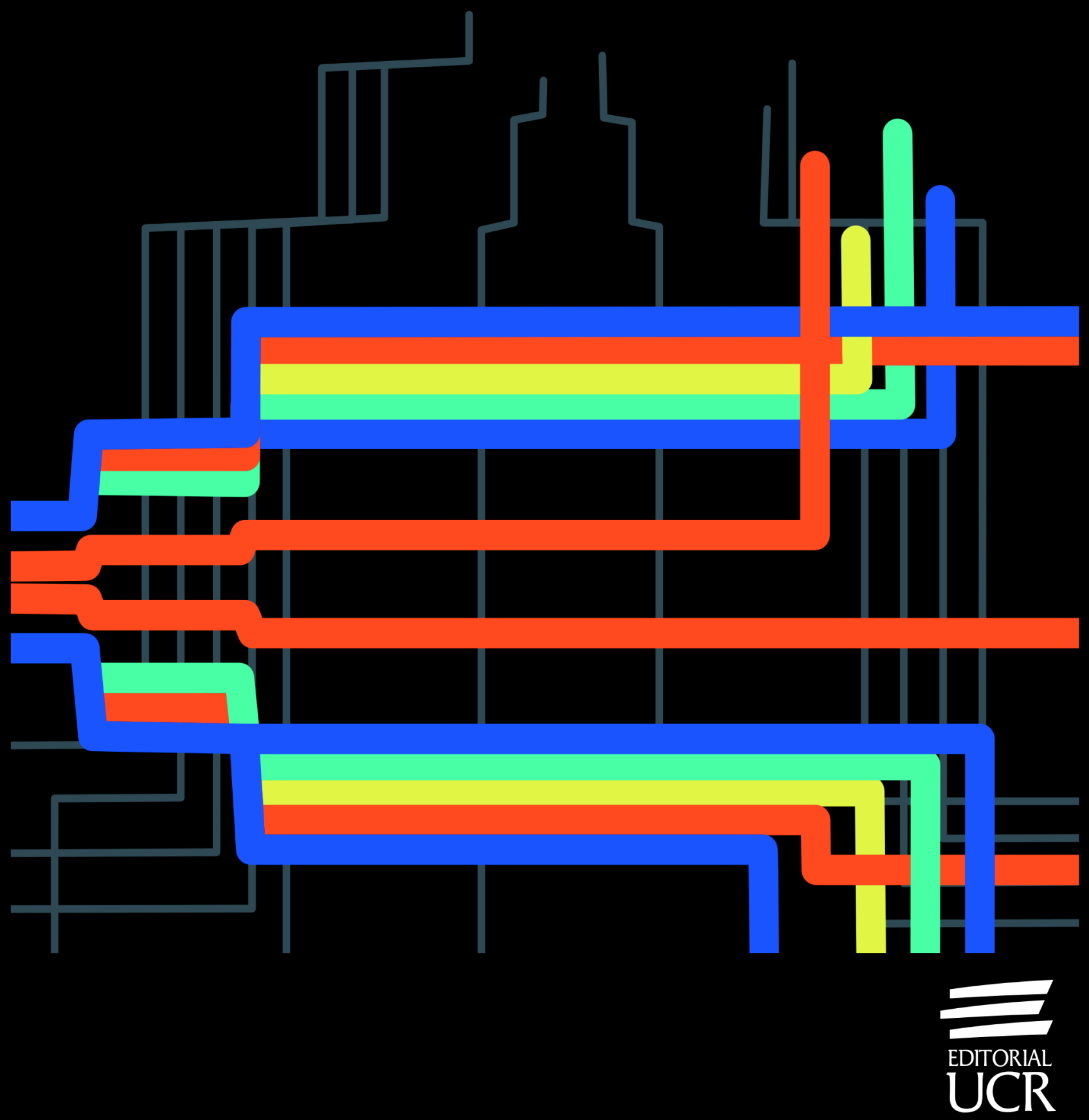




\title{
Metodología FMECA para un aerogenerador doblemente alimentado
}

\section{FMECA methodology for a doubly-fed wind turbine}

\author{
Andrey Solano Artavia \\ Escuela de Ingeniería Eléctrica \\ Universidad de Costa Rica, San José, Costa Rica \\ Email: andrey.solano@ucr.ac.cr \\ ORCID: 0000-0002-2463-8338 \\ Brandon Cruz Jiménez. \\ Escuela de Ingeniería Eléctrica \\ Universidad de Costa Rica, San José, Costa Rica \\ Email:brandon.cruz@ucr.ac.cr \\ ORCID: 0000-0001-9353-261X \\ Cristian Ramos Fernández \\ Escuela de Ingeniería Eléctrica \\ Universidad de Costa Rica, San José, Costa Rica \\ Email:cristian.ramosfernadez@ucr.ac.cr \\ ORCID: 0000-0002-4410-7577 \\ Kevin Solís Guzmán \\ Escuela de Ingeniería Eléctrica \\ Universidad de Costa Rica, San José, Costa Rica \\ Email:kevin.solisguzman@ucr.ac.cr \\ ORCID: 0000-0002-8621-0839 \\ Óscar Núñez Mata \\ Escuela de Ingeniería Eléctrica \\ Universidad de Costa Rica, San José, Costa Rica \\ Email: oscar.nunezmata@ucr.ac.cr \\ ORCID: 0000-0002-5410-6121 \\ Aramis Pérez Mora \\ Escuela de Ingeniería Eléctrica \\ Universidad de Costa Rica, San José, Costa Rica \\ Email:aramis.perez@ucr.ac.cr \\ ORCID: 0000-0002-6783-2659
}

Recibido: 5 de marzo $2021 \quad$ Aceptado: 7 de junio 2021 


\title{
Resumen
}

Debido a la complejidad de los sistemas eólicos, la necesidad de contar con aerogeneradores fiables es de suma importancia, por ello, se han desarrollado metodologías para mejorar sus características y desempeño. El método de análisis de modos de falla, efectos y criticidad se ha utilizado ampliamente para estudiar la confiabilidad de múltiples sistemas y procesos en diversas áreas, entre las cuales se encuentra la de generación de energía eléctrica. En este documento se presenta la aplicación de dicha metodología a un aerogenerador de inducción doblemente alimentado, para realizar una evaluación cualitativa y cuantitativa de las características de falla en el mismo. Además, para tomar en cuenta consideraciones económicas en el análisis, se presenta el enfoque del método basado en el riesgo, con el fin de mitigar las limitaciones que presenta el método tradicional. Como resultado, se determina que las estrategias de mantenimiento deben enfocarse principalmente en el generador, luego el sistema eléctrico y posteriormente en las aspas.

\section{Palabras clave:}

Aerogenerador, Criticidad, FMECA, Modo de falla, Prevención de fallas catastróficas, Reducción de costos.

\begin{abstract}
Due to the complexity of wind systems, the need for reliable wind turbines becomes highly relevant, therefore methodologies have been developed to improve their characteristics and performance. The failure modes, effects and criticality analysis method has been used to study the reliability of multiple systems and processes in various areas, among which is the electrical power generation. This document presents the application of this methodology to a doubly fed induction wind turbine, to perform a qualitative and quantitative evaluation of its failure characteristics. In addition, to take into account economic considerations in the analysis, a risk-based approach is presented to mitigate the limitations presented by the traditional method. As a result, it is determined that maintenance strategies should focus primarily on the generator, then the electrical system, and later on the blades.
\end{abstract}

Keywords:

Wind turbine, Criticality, FMECA, Failure mode, Failure prevention, Cost reduction. 


\section{INTRODUCCIÓN}

Debido al auge de las energías renovables en las últimas décadas, distintas industrias como la generación eólica han experimentado un crecimiento exponencial. Por la intermitencia inherente en la generación eólica, la fiabilidad de los aerogeneradores se vuelve esencial en un entorno tan competitivo como el mercado energético. En este sentido, la optimización del mantenimiento toma un papel indispensable para aumentar la disponibilidad de los aerogeneradores, sin embargo, se debe determinar cuál de los subsistemas requiere una mayor atención, pues una alta disponibilidad no necesariamente corresponde a una correcta gestión de mantenimiento, sino a un servicio rápido en caso de falla [1].

No obstante, en Costa Rica los operadores de parques eólicos no cuentan con análisis que brinden información sobre las consecuencias en el proceso de generación de energía eléctrica, en caso de que un elemento del aerogenerador llegase a fallar, lo que reduce la capacidad y la rapidez de invertir recursos en el mantenimiento de elementos críticos y de riesgo para el sistema. Esto conduce a estrategias de mantenimiento insuficientes que afectan la disponibilidad de los aerogeneradores y provocan que los costos de mantenimiento sean elevados.

Por ello, es necesario realizar análisis que permitan jerarquizar los elementos del aerogenerador en función de su impacto al proceso de conversión de energía, de esta forma, determinar qué elementos presentan mayor riesgo para el sistema, para así dirigir el esfuerzo y los recursos a las áreas donde sea más urgente mejorar la fiabilidad, lo que permitirá establecer programas de mantenimiento óptimos con el fin de minimizar costos y aumentar la disponibilidad de los aerogeneradores [2].

En este contexto, el concepto de riesgo se ha utilizado para priorizar sistemas mediante la evaluación de escenarios que se pueden presentar en la operación del equipo, esto al determinar la probabilidad de que un evento específico ocurra y las consecuencias que este conlleva, y así poder categorizarlas. Existen diferentes métodos que se pueden aplicar para priorizar un sistema ante un escenario de falla; el Análisis de Modos y Efectos de Falla (FMEA, por sus siglas en inglés) es una técnica para el estudio de los escenarios de falla en el contexto de operación de activos físicos, consiste en un proceso lógico de evaluación de modos de falla del proceso, sus causas y efectos. Además, se puede ampliar el método al aplicar un análisis de criticidad para estimar la probabilidad y gravedad de cada modo de falla, a este método se le conoce como Análisis de Modos de Falla, Efectos y Criticidad (FMECA, por sus siglas en inglés) [3].

El método FMEA y su ampliación FMECA, se han utilizado ampliamente en la industria eólica. La metodología FMEA fue utilizada por [4] en la evaluación de una turbina eólica de 2 MW con un generador de inducción doblemente alimentado. Este identificó más de 150 componentes de una turbina eólica, que se han usado como base para el desglose de turbinas eólicas en muchos estudios. Al comparar los resultados cuantitativos obtenidos del FMEA y los datos de campo de confiabilidad de turbinas eólicas, concluyó que el FMEA puede servir como herramienta preliminar de predicción de las tasas de falla. Entonces, esta puede ser una herramienta útil para 
identificar puntos débiles en el diseño de turbinas eólicas y en la optimización de operación y mantenimiento del parque eólico [4].

Posteriormente, [5] identificó las limitaciones de un FMEA tradicional cuando se aplica en la evaluación de turbinas eólicas. Por ello, presenta un enfoque cuantitativo llamado FMEA basado en riesgos, que utiliza las probabilidades de falla y los costos incurridos. Sus resultados muestran que la definición de los modos de falla en función de su contribución al costo total de falla es más realista y práctica que el enfoque común de FMEA [5].

Por otra parte, [6] aplicó FMEA a un aerogenerador de 5 MW para evaluar las características de confiabilidad de las turbinas eólicas. Con el estudio se identificaron áreas susceptibles de fallas, tales como las aspas y el sistema de lubricación, y destacó aquellas con potencial de tener instalados sistemas de monitoreo de condición para permitir un mantenimiento efectivo. Además, concluyó que la introducción de procedimientos como este permite incrementar el tiempo medio entre fallas de las turbinas, proporcionando una mayor eficiencia [6].

Así mismo, para realizar un análisis de confiabilidad a turbinas eólicas marinas flotantes, [7] aplicó un método de FMEA modificado denominado correlación FMEA, que estudia la conexión entre los modos de falla y su efecto en la probabilidad de falla a todo el sistema. El estudio reveló que la estructura de los cimientos y el sistema de amarre eran sistemas de alta relevancia, con las consecuencias de falla potencialmente más graves. Con este método los autores ofrecen la posibilidad de que el mismo pueda adaptarse a aplicaciones prácticas para instruir u optimizar el proceso de diseño del sistema de turbinas eólicas marinas flotantes y reducir los costos de reparación [7].

Este trabajo muestra la aplicación de la metodología FMECA a un aerogenerador doblemente alimentado, con el fin de obtener una jerarquización de los componentes del aerogenerador en función del impacto en el proceso de generación de energía, el costo y criticidad en caso de ocurrir una falla catastrófica y así determinar cuál elemento representa un mayor riesgo para la operación del sistema. Se espera que dicha jerarquización pueda ser utilizada por los operadores de parques eólicos en el país en la optimización de planes de mantenimiento y la toma de decisiones informadas, que les permitan ser más competitivos en el entorno de la generación de energía eléctrica.

A tal efecto, en la sección II de este artículo se describe de forma general el aerogenerador utilizado para el caso de estudio y el fundamento teórico del método FMECA, posteriormente, en la sección III se presenta la aplicación del método a un aerogenerador, por último, en las secciones IV y V se muestran los resultados obtenidos y las conclusiones, respectivamente.

\section{MARCO TEÓRICO}

\subsection{Descripción del aerogenerador}

La energía eólica es una de las fuentes con mayor perspectiva para la generación eléctrica a partir de recursos renovables, por eso, el porcentaje de capacidad instalada de aerogeneradores 
crece cada año [8]. El aerogenerador es un dispositivo destinado a extraer la energía utilizable del viento. Cuando el viento pasa por las aspas (también llamadas palas) del aerogenerador, parte de su energía cinética gira las mismas, transformándola en energía mecánica, con una velocidad de rotación más baja a una velocidad de rotación más alta a través de la caja multiplicadora para que, posteriormente, el generador convierta esta rotación en energía eléctrica [9], [10]. En la Fig. 1 se muestra la distribución de los principales componentes de un aerogenerador tipo 3.

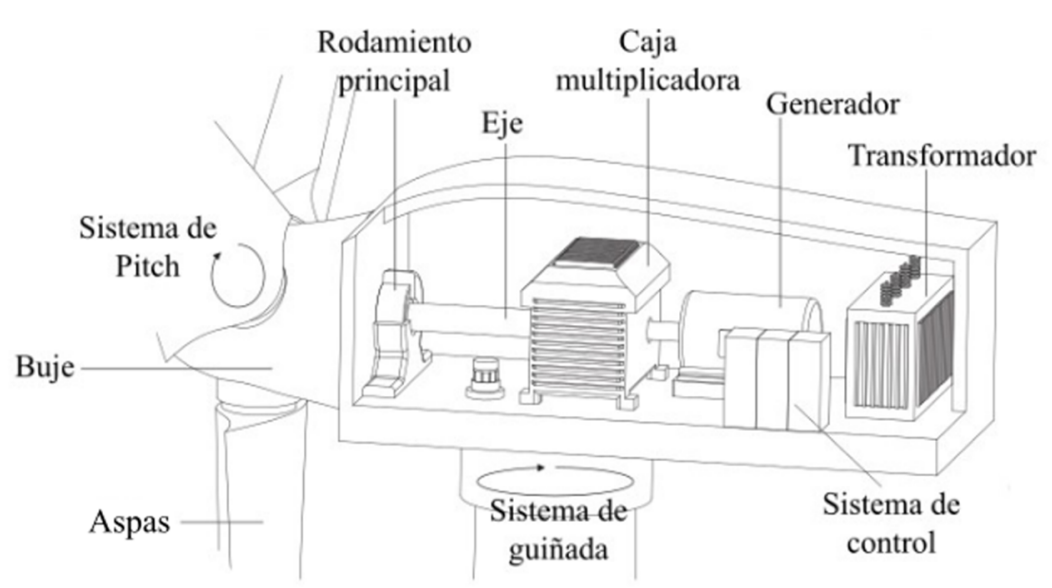

Fig. 1. Componentes principales de un aerogenerador tipo 3. Fuente: Adaptado de [3].

\subsection{Detección de fallas}

Los componentes de un aerogenerador están sujetos a fallas, debido a las condiciones de operación y de su entorno. Estos pueden sufrir una falla, definida como una variación en su estructura o en sus parámetros que limitan su funcionamiento nominal, mas no implica una inhabilitación para operar. Por otra parte, se puede presentar una falla catastrófica en el elemento, la cual implica la incapacidad del mismo para operar [11].

La implementación de sistemas de detección de fallas tiene como objetivo evitar daños catastróficos en los componentes del sistema y facilitar el desarrollo de estrategias eficientes de reparación y mantenimiento. Cuando se detecta una anomalía se debe realizar un diagnóstico para identificar el tipo, ubicación y criticidad de la falla, con el fin de aplicar la acción de mantenimiento adecuada.

Las fallas en los aerogeneradores pueden ocurrir a nivel de componente o a nivel de sistema, por ello, es necesario monitorear las fallas a nivel de componente, pues estas se pueden propagar en el sistema e influir en los demás componentes, así una falla en un componente de menor importancia puede llegar a afectar todo el sistema y eventualmente ocasionar que el sistema quede fuera de operación [11].

Con un esquema de gestión de mantenimiento efectivo que involucra detección, diagnóstico y pronóstico de fallas, y planes de mantenimiento basados en el análisis de datos, se obtienen algunas ventajas, como [12]: 
1) Evitar fallas prematuras, fallas catastróficas o propagación de fallas menores en el sistema.

2) Reducir costes de mantenimiento, pues los programas de mantenimiento adecuados evitan acciones innecesarias.

3) Recopilar detalles importantes sobre el comportamiento de los aerogeneradores en el tiempo.

4) Proporcionar información al operador del parque eólico necesaria para mejorar la eficiencia operativa, asistiendo al proceso de toma de decisiones.

Existen diferentes tipos de esquemas para la detección de fallas, como la redundancia de hardware, enfoque basado en modelos, enfoque basado en análisis de señales, entre otros. Un enfoque basado en modelos se implementa con una representación del proceso programada en un computador, donde se modela cualitativa o cuantitativamente el comportamiento dinámico y el desempeño en régimen permanente. La diferencia entre las variables del proceso medidas por los diferentes sensores y sus estimaciones obtenidas mediante el modelo se denomina valor residual [11]. Entonces, el valor residual es utilizado como indicador de falla, pues representa la desviación entre las mediciones y los cálculos basados en la ecuación del modelo, con el cual se detecta una falla cuando dicho valor cruza un umbral predefinido [13].

En el enfoque basado en análisis de señales, se parte del supuesto de que las señales del proceso transportan información sobre las fallas de interés, donde es posible la detección de fallas mediante un procesamiento de señal adecuado. Los síntomas típicos son funciones en el dominio del tiempo como magnitudes, valores medios aritméticos o cuadráticos, tendencias o funciones en el dominio de la frecuencia como densidades de potencia espectral, líneas espectrales de frecuencia, entre otros. En este enfoque se encuentran el análisis de señales eléctricas y el análisis de vibraciones. El análisis mediante señales eléctricas tiene la ventaja que estas son medidas desde los terminales del generador, por lo que no necesita la instalación de equipo adicional, lo que resulta en un enfoque poco intrusivo [11]. En cuanto al análisis de vibraciones, este se usa principalmente para la detección de fallas en rodamientos, utilizando sensores de vibración como acelerómetros o sensores de desplazamiento [14].

La mayoría de esquemas utilizados para la detección de fallas, requieren datos específicos que provienen de distintos sensores y dispositivos de adquisición de datos. Estos datos de funcionamiento y valores de medición, además de ser utilizados para la detección de fallas, deben ser almacenados y estar disponibles para análisis posteriores, como el presentado en este documento. Sin embargo, para poder realizar análisis más sólidos se requieren varios grupos de datos, como los mencionados anteriormente: datos del equipo, datos de mantenimiento e inspección, datos de fallas e información de costos [15].

El problema es que generalmente los datos se encuentran dispersos en diferentes bases de datos o no existen, como lo destaca [16] en su investigación, donde se señala que en Costa Rica no se cuenta con datos históricos de fallas y tiempos de paro de los aerogeneradores, por lo que se debe recurrir a bases de datos internacionales. Esto genera dificultades para el análisis posterior y 
la toma de decisiones, por ello, es necesaria la creación de bases de datos consistentes y uniformes, que contengan los diferentes parámetros utilizados, facilitando el análisis posterior y el proceso de seguimiento [17].

\subsection{Análisis de modos de falla, efectos y criticidad}

El FMECA es una metodología que tiene como objetivo la identificación de los modos de falla que representan alto riesgo para el proceso analizado. Para comprender con mayor detalle el método, se debe entender por modos de falla las posibles formas en las cuales un elemento puede llegar a fallar, y por efecto de falla, las consecuencias que puede generar un determinado modo de falla en caso de que no se realice ningún procedimiento predictivo o preventivo [18].

El desarrollo de un FMECA consta de dos partes principales: una cualitativa y una cuantitativa. En la parte cualitativa, el sistema o proceso a analizar se descompone en todas sus partes constituyentes, y se estudian los modos de falla que pueden ocurrir en cada uno para así determinar sus posibles efectos. Se delimita el nivel de estudio de los elementos de interés, y se detallan sus funciones, sus posibles fallas funcionales y las consecuencias o efectos que pueden generar sobre el elemento y el sistema general.

Por otro lado, la parte cuantitativa consta de la asignación de valores numéricos a los índices de severidad $\left(I_{S}\right)$, que indica qué tan grave es la falla presentada, el índice de ocurrencia $\left(I_{O}\right)$, que indica con qué frecuencia se presenta una falla en el sistema o elemento y el índice de detección $\left(I_{D}\right)$, que indica qué tan probable es localizar la falla del sistema o elemento. Estos índices son asignados a los elementos de estudio o propiamente a los modos de falla identificados. A partir de los índices asignados es posible calcular el Índice de Prioridad de Riesgo (IPR), que indica cuál de los elementos o modos de falla en el proceso representa un mayor riesgo. Su cálculo se realiza mediante (1):

$$
I P R=I_{S} \cdot I_{O} \cdot I_{D}
$$

Una problemática del FMECA se origina debido a que el IPR obtenido, por sí solo, no distingue entre modos de falla de severidad alta y baja probabilidad de ocurrencia, o un modo de falla menos severo con mayor probabilidad de ocurrencia. Para solventar el problema mencionado, existe un enfoque que incorpora el costo asociado con cada modo de falla, llamado FMECA-Basado en el Riesgo (FMECA-BR) [5]. Este análisis permite evaluar el riesgo de cada modo de falla, al cual se le llama Número de Prioridad de Costo, $C P N$ (del inglés “Cost Priority Number"), dado en unidades monetarias. El mismo se calcula con (2), donde $P_{F}$ es la probabilidad de ocurrencia, $P_{N D}$ la probabilidad de no detectar la falla y $C_{F}$ el costo de la falla:

$$
C P N=P_{F} \cdot P_{N D} \cdot C_{F}
$$

El valor de $P_{F}$ se extrae de los datos históricos, específicamente de la ocurrencia del modo de falla en cuestión del complejo eólico. La $P_{N D}$ se determina de acuerdo a (3), donde NF es el número de fallas reales y $N_{F V}$ el número total de vulnerabilidades de falla, que se definen como la suma de la cantidad de fallas reales y la cantidad de posibles fallas detectadas antes de que ocurran, mediante monitoreo, inspección o mantenimiento. El $C_{F}$ se determina mediante (4), este incluye el costo de 
las piezas que deben reemplazarse $\left(C_{P}\right)$, el costo del servicio $\left(C_{S}\right)$ y el costo de oportunidad $\left(C_{O}\right)$, siendo así la suma de los ingresos que el propietario del parque eólico recibiría por la venta de energía, en caso de que no ocurriera la falla; así como el costo total de trabajo adicional requerido para la reparación $\left(C_{L}\right)[5]$ :

$$
\begin{aligned}
& P_{N D}=N_{F} / N_{F V}, \\
& C_{F}=C_{P}+C_{S}+C_{O}+C_{L} .
\end{aligned}
$$

El $C_{O}$ se determina con (5), donde $D_{F}$ es la duración de la falla, $W P_{o u t}$ es la potencia promedio de salida y EPR es la tasa promedio de compra de energía dentro de esta duración. El $C_{L}$ se determina con (6), donde $N C$ y $M H R$ representan el número de equipos de reparación y el precio que representa la mano de obra, respectivamente:

$$
\begin{aligned}
& C_{O}=D_{F} \cdot W P_{\text {oUT }} \bullet E P R, \\
& C_{L}=D_{F} \cdot N C \cdot M H R .
\end{aligned}
$$

Una vez determinado el número de prioridad de costo, se puede utilizar para calcular el costo total anual de falla $(T F C)$ del sistema para cualquier duración específica de interés $\left(D_{\text {int }}\right)$. El costo total de falla se determina con (7), donde "m" representa el número total de modos de falla y $N F V(\mathrm{i}$, $D_{\text {int }}$ ) denota el número de vulnerabilidades de falla para cada modo de falla.

$$
T F C=\sum_{i=1}^{m} N_{F V}\left(i, D_{i n t}\right) \cdot C P N
$$

\section{ANÁLISIS FMECA A UN AEROGENERADOR}

Para la elaboración del análisis cualitativo, se realiza una descomposición de primer nivel, es decir, se detallan todos los elementos principales que componen el sistema, que en el caso del aerogenerador corresponden a 12 elementos en los cuales se centró el estudio. El siguiente paso consiste en detallar las posibles fallas funcionales y las posibles consecuencias o efectos de tales fallas para cada uno de los elementos. Finalmente, se detallan todos los posibles modos de falla para cada elemento, así como todas sus posibles causas.

Una vez que se tienen delimitados los elementos de estudio, se procede a realizar un análisis cuantitativo de dichos elementos, el cual consiste en la asignación de los índices de severidad, ocurrencia y detección. Para la asignación de estos tres índices, se tomaron los datos de fallas reales proporcionados por [1], en donde se realiza un recuento de las fallas ocurridas en aerogeneradores onshore de Suecia, Alemania y Finlandia, entre los años 1997 y el 2005. En este estudio se contabilizan las fallas ocurridas en aerogeneradores con potencias entre los $490 \mathrm{~kW}$ y los $1500 \mathrm{~kW}$.

La asignación de los índices se realiza de la siguiente forma:

- $\quad$ Para el índice de severidad, se toma la cantidad de horas en baja por año debido a cada uno de los elementos. Con esto, se le asigna un índice de 10 a aquel elemento que provoque una mayor cantidad de horas en baja, y usando este como referencia, son asignados los índices 
para los otros elementos. De esta forma, se asigna un índice de severidad de 10 al sistema de control, y un índice de 1 al freno, el cual ocasiona cerca de un $10 \%$ de las horas en baja que ocasiona la falla del sistema de control.

- $\quad$ Para el índice de ocurrencia, se toma la cantidad de fallas presentadas por año en cada uno de los elementos del sistema, y de manera similar al procedimiento anterior, se le asigna un índice de 10 a aquel elemento que presente más fallas, en este caso el sistema eléctrico. De forma similar al índice de severidad, se utiliza este elemento con índice de 10 para asignar los índices de los otros elementos; así, por ejemplo, al sistema hidráulico le es asignado un índice de 8 debido a que presenta cerca del $80 \%$ de las fallas por año que presenta el sistema eléctrico.

- $\quad$ El caso del índice de detección es distinto a los índices anteriores, dado que no es respaldado por ningún tipo de medición o cuantización, sino que es asignado de manera subjetiva en base a técnicas de monitoreo de fallas y a la facilidad con la que es detectada la falla; por esta razón, se toman los índices de detección presentados en [5] como base para la realización del análisis.

Asignados los índices para cada uno de los elementos, se procede a realizar el cálculo del IPR mediante (1), para luego establecer una jerarquización de los elementos del aerogenerador según su nivel de riesgo, pues entre mayor sea su $I P R$, mayor es su impacto en la seguridad y/o un buen funcionamiento del aerogenerador. Existen distintas maneras de realizar una jerarquización de este tipo, y esta dependen enteramente del criterio de la persona a cargo de realizar el análisis. En el caso de este documento, se propone una regla de jerarquización por intervalos, en la cual, el nivel de riesgo de un elemento se categoriza en uno de 6 posibles niveles en base a su IPR y el intervalo de valores en los que este se encuentre. Buscando una distribución lo más uniforme posible de los elementos dentro de las 6 categorías de riesgo, se definen los límites de estos intervalos a criterio de los autores. En la Tabla I, se muestra la regla de jerarquización utilizada para categorizar el riesgo de una falla en los distintos elementos del aerogenerador basado en su IPR.

TABLA I

CATEGORIZACIÓN DEL RIESGO SEGÚN IPR

\begin{tabular}{lc}
\hline \multicolumn{1}{c}{ Categoría de riesgo } & IPR \\
\hline Crítico & $350<$ \\
Muy alto & $200<\& \leq 350$ \\
Alto & $100<\& \leq 200$ \\
Moderado & $50<\& \leq 100$ \\
Bajo & $10<\& \leq 50$ \\
Muy bajo & $\leq 10$ \\
\hline
\end{tabular}

Una vez calculado el $I P R$ para cada elemento y llevar a cabo la jerarquización, se procede a realizar el análisis de costos, determinando primero $P_{F}$ y $P_{N D}$ con los datos de cantidades de fallas reales presentes en [5]. Partiendo de las probabilidades de ocurrencia y las probabilidades de que no se detecten fallas para el elemento respectivo, se procede a calcular o consultar los costos de servicio, costos de la mano de obra y el costo de oportunidad que representa la ocurrencia de la 
falla. Una vez obtenidos los datos de fallas de bases datos reales o datos estimados basándose en referencias, se procede a determinar la duración de la falla, y posteriormente calcular su costo total para cada modo de falla. Cabe destacar que los costos de las partes del aerogenerador que se deben reemplazar se obtienen de un parque eólico costarricense, con el fin de apegar el estudio lo más posible a un caso real. Este proceso se puede extrapolar a otros tipos de aerogenerador debido a que la información necesaria para el análisis no es específica, es basada en costos que se presentan en cualquier tipo de máquina. Por último, se deberá calcular el $C P N$ para cada elemento del aerogenerador, con el fin de priorizar ciertos subsistemas mediante la comparación de los $C P N$ obtenidos.

\section{RESULTADOS}

De la metodología FMECA descrita en la sección anterior, se obtiene una descripción detallada de los 12 elementos principales del aerogenerador, sus funciones principales dentro del sistema y las implicaciones más importantes que tendría el sistema al presentarse una falla en dichos elementos; además, se identifica un total de 24 modos de falla y 61 posibles causas.

Con respecto al análisis cuantitativo, se realiza la asignación de los índices de severidad, ocurrencia y detección para cada uno de los elementos del estudio siguiendo la metodología descrita, y posteriormente se realiza el cálculo del $I P R$ y la jerarquización en base al mismo, según la regla de jerarquización utilizada. Finalmente, en la Tabla II se muestran los resultados obtenidos en el análisis cualitativo del aerogenerador.

TABLA II

JERARQUIZACIÓN DE RIESGO PARA LOS ELEMENTOS DEL AEROGENERADOR

\begin{tabular}{lccccc}
\hline \multicolumn{1}{c}{ Elemento } & Severidad & Ocurrencia & Detección & IPR & Riesgo \\
\hline Sistema de control & 10 & 8 & 7 & 560 & Crítico \\
Multiplicadora & 10 & 6 & 7 & 420 & Crítico \\
Sistema eléctrico & 8 & 10 & 4 & 320 & Muy alto \\
Aspas & 5 & 8 & 4 & 160 & Alto \\
Sistema de guiñada & 7 & 4 & 4 & 112 & Alto \\
Sensores & 3 & 9 & 4 & 108 & Alto \\
Sistema hidráulico & 3 & 8 & 4 & 96 & Moderado \\
Generador & 5 & 4 & 4 & 80 & Moderado \\
Tren de impulsión & 2 & 1 & 7 & 14 & Bajo \\
Freno & 1 & 1 & 7 & 7 & Muy bajo \\
Estructura & 1 & 1 & 7 & 7 & Muy bajo \\
Buje & 1 & 1 & 4 & 4 & Muy bajo \\
\hline
\end{tabular}

Es importante resaltar que, aunque un elemento presente un alto $I P R$, no representa, necesariamente, que las fallas se presenten de manera frecuente, o bien, que estas sean críticas para el sistema. Por lo tanto, puede que se obtenga un alto valor de $I P R$, basado en una baja ocurrencia y una alta criticidad, como sería el caso de la multiplicadora, la cual no tiene un índice de ocurrencia entre 
los más altos, sin embargo, la severidad de presentarse una falla es la más elevada junto con el sistema de control. Por otro lado, se puede obtener un alto valor de IPR basado en una alta ocurrencia y una baja criticidad, como se refleja en los sensores, los cuales presentan una severidad baja pero la ocurrencia de una falla en ellos es bastante alta, lo que ocasiona el aumento en el valor del IPR. Esta es la principal debilidad del FMECA, tal y como se explica en la nota teórica, dado que no es posible extraer esa información tan sólo del análisis del IPR. Para contrarrestar esta debilidad, se lleva a cabo el FMECA Basado en Riesgo.

Para iniciar el análisis FMECA-BR, se calculan los costos monetarios de cada subsistema en base a precios de un aerogenerador en Costa Rica, con el fin de considerar las pérdidas económicas por venta de energía para los periodos de punta, valle y nocturno, mientras el sistema está de baja. De esta forma, se contabilizan los costos de producción que influyen negativamente sobre los costos totales de la falla. Esto se realiza tomando en cuenta las tarifas vigentes para el mes de julio del año 2020, definidas en [19]. Al realizar el análisis de costos al aerogenerador se obtiene el $C P N$ para cada elemento, donde se evidencia que los elementos más críticos son: el generador, el tren de impulsión y el sistema eléctrico, como se muestra en la Tabla III.

TABLA III

CÁlCULO DE COSTOS DE FALLA MÁS SIGNIFICATIVOS. DATOS TOMADOS DE [3]

\begin{tabular}{lccccccc}
\hline \multicolumn{1}{c}{ Subsistema } & $\mathbf{D}_{\mathbf{F}}[$ horas $]$ & $\mathbf{C}_{\mathbf{P}}[\mathbf{\$}]$ & $\mathbf{C}_{\mathbf{S}}[\mathbf{\$}]$ & $\mathbf{C}_{\mathbf{L}}[\mathbf{\$}]$ & $\mathbf{C}_{\mathbf{0}}[\mathbf{\$}]$ & $\mathbf{C}_{\mathbf{F}}[\mathbf{\$}]$ & $\mathbf{C P N}[\mathbf{\$}]$ \\
\hline Generador & 180 & 61014 & 29325 & 180 & 3529,25 & 94048,25 & 13035,09 \\
Tren de impulsión & 120 & 9827 & 14700 & 120 & 2352,83 & 26999,83 & 4373,97 \\
Sistema eléctrico & 100 & 11319 & 1000 & 6000 & 1960,70 & 20279,70 & 2798,60 \\
Aspas & 96 & 37125 & 18375 & 960 & 1882,27 & 58342,27 & 2654,57 \\
Estructura & 96 & 27300 & 22050 & 960 & 1882,27 & 52192,27 & 422,76 \\
Buje & 72 & 4800 & 1800 & 720 & 1411,70 & 8731,70 & 322,20 \\
\hline
\end{tabular}

El CPN aplica para una única falla en cada subsistema, por lo cual, no contempla la repetitividad de fallas en un periodo anual. Por eso, se complementa con el Costo Total de Falla (TFC), el cual determina el coste final debido a todas las ocurrencias anuales en un mismo elemento. Como ejemplo, para el generador el $C P N$ es de $\$ 13055,09$ y al año el TFC de ese elemento corresponde a $\$ 172063,34$, de ahí la necesidad de priorizar de mayor a menor costo los elementos y proyectar a futuro las inversiones necesarias para anticipar la logística relacionada a la reparación o mantenimiento de cada subsistema.

Finalmente, se comparan los resultados de los $C P N$ junto a los IPR para cada subsistema, esto permite contemplar los elementos más críticos del sistema y los costos de las fallas que se pueden presentar, como se observa en la Fig. 2. 


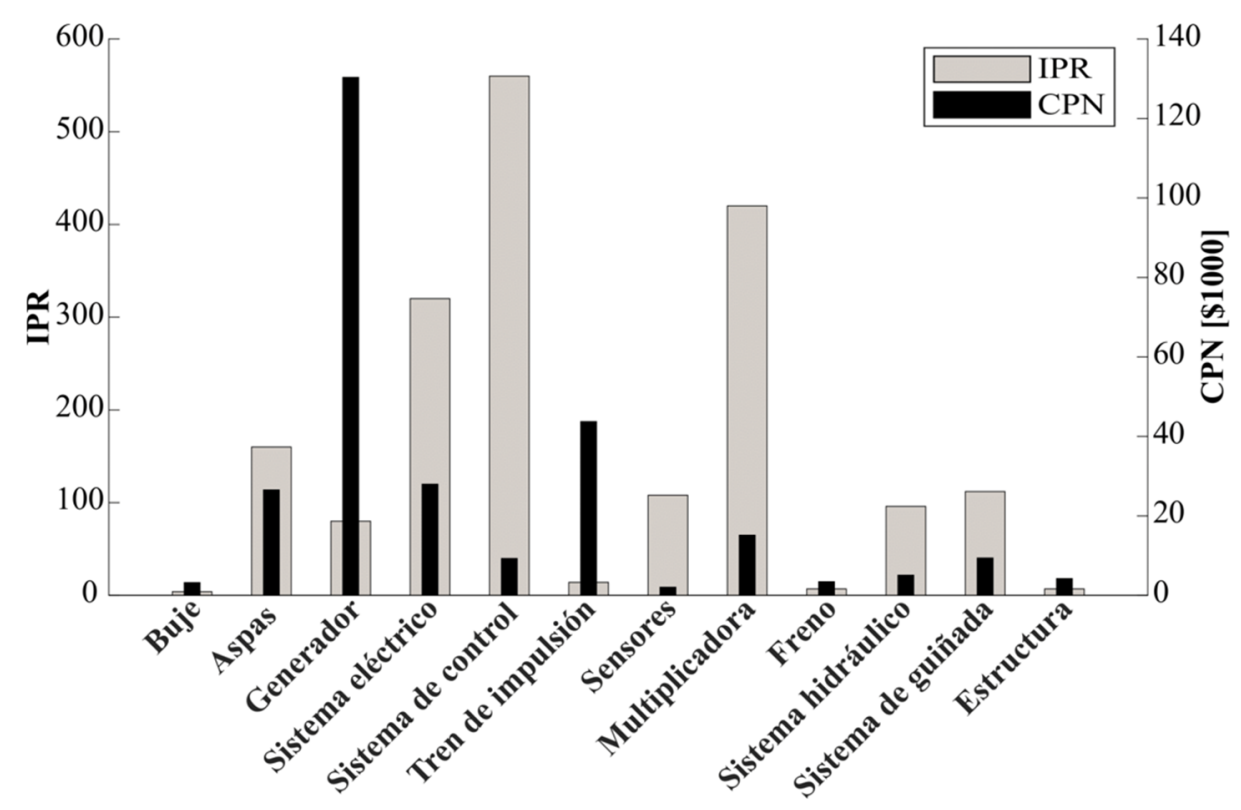

Fig. 2. $I P R$ y $C P N$ para las partes analizadas.

\section{CONCLUSIONES}

El FMECA es una metodología simple que permite entender cómo funciona un sistema y cómo puede llegar a fallar, esto ayuda a enfocar los esfuerzos de monitoreo con el fin de detectar y posteriormente pronosticar las fallas, para que así las estrategias de mantenimiento funcionen de manera optimizada, de esta forma, minimizar el tiempo de inactividad y disminuir el costo de la falla. Aparte, mencionar que un componente con índice de ocurrencia alto no quiere decir que sea el más severo, pues la accesibilidad, las formas de corregir el problema, los costos de equipo y transporte hacen que estos dos no tengan gran dependencia el uno del otro.

Finalmente, la jerarquización del riesgo basado en $I P R$ y en $C P N$ es la manera completa de aplicar el método, pues muestra qué tan crítico es un componente y qué tan rápido hay que atenderlo, para invertir en los subsistemas con alta criticidad, que permita potenciar la fiabilidad del complejo.

\section{AGRADECIMIENTOS}

Esta investigación se hizo bajo el proyecto de investigación "Detección de fallas, control e integración de sistemas de energías renovables no convencionales con almacenamiento energético para redes inteligentes" código 322-C1-467 de la Universidad de Costa Rica. 


\section{REFERENCIAS}

[1] J. Ribrant y L. M. Bertling, "Survey of failures in wind power systems with focus on Swedish wind power plants during 1997-2005", IEEE Transactions on Energy Conversion, vol. 22, no. 1, pp. 167173, marzo, 2007. DOI: 10.1109/TEC.2006.889614

[2] J. M. Gonzáles, L. Amendola y T. Depool, "Modelo de criticidad operacional en generadores de parques eólicos", presentado en XII Congreso Internacional de Ingeniería de Proyectos, Zaragoza, España, 2008, pp. 2331-2340.

[3] M. N. Scheu, L. Tremps, U. Smolka, A. Kolios y F. Brennan, “A systematic Failure Mode Effects and Criticality Analysis for offshore wind turbine systems towards integrated condition based maintenance strategies", Ocean Engineering, vol. 176, pp. 118-133, marzo, 2019. DOI: https://doi. org/10.1016/j.oceaneng.2019.02.048

[4] H. Arabian-Hoseynabadi, H. Oraee y P.J. Tavner, "Failure Modes and Effects Analysis (FMEA) for wind turbines", International Journal of Electrical Power \& Energy Systems, vol. 32, no. 7, pp. 817-824, septiembre, 2010. DOI: https://doi.org/10.1016/j.ijepes.2010.01.019.

[5] S. Kahrobaee y S. Asgarpoor. "Risk-based failure mode and effect analysis for wind turbines (rbfmea)", presentado en 2011 North American Power Symposium, Boston, Estados Unidos, agosto, 2011, pp. 1-7.

[6] M. Gauravkumar Bharatbhai, "Failure Mode and Effect Analysis of Repower 5M Wind Turbine", International Journal of Advance Research in Engineering, Science \& Technology, vol. 2, no. 5, pp. 1-8, mayo, 2015.

[7] J. Kang, L. Sun, H. Sun y C. Wu, "Risk assessment of floating offshore wind turbine based on correlation-FMEA", Ocean Engineering, vol. 129, pp. 382-388, enero, 2017. DOI: https://doi. org/10.1016/j.oceaneng.2016.11.048.

[8] N. V. Zubova y A. A. Achitaev, "Application of Neuro-Fuzzy Control Systems for Increasing the Energy Efficiency of Wind Turbines", presentado en XIV International Scientific-Technical Conference on Actual Problems of Electronics Instrument Engineering (APEIE), Novosibirsk, Rusia, octubre 2-6, 2018, pp. 518-521, DOI: https://doi.org/10.1109/APEIE.2018.8546210.

[9] M. F. Ahmed Fayeem, A. H. Galib y P. Saha, "Micro Wind Turbine as an Alternative Power Source in Bangladesh", presentado en 2019 International Conference on Sustainable Technologies for Industry 4.0 (STI), Dhaka, Bangladesh, diciembre 24-25, 2019, pp. 1-4, DOI: https://doi.org/10.1109/ STI47673.2019.9067980.

[10] Y. Li, Y. Zheng, N. Zhu y F. Zhao, "Wind Turbine Kinetic Energy Accumulation and Release Regulation for Wind Farm Optimization", presentado en 2019 4th International Conference on Mechanical, Control and Computer Engineering (ICMCCE), Hohhot, China, Octubre 24-26, 2019, pp. 2312314, DOI: https://doi.org/10.1109/ICMCCE48743.2019.00060

[11] S. Pourmohammad y A. Fekih. "Fault tolerant control of wind turbine system - a review", presentado en 2011 IEEE Green Technologies Conference (IEEE-Green), Baton Rouge, Estados Unidos, abril 14-15, 2011, pp. 1-6, DOI: https://doi.org/10.1109/GREEN.2011.5754880

[12] F. P. García y M. Papaelias, "An overview of wind turbine maintenance management", en Nom-Destructive Testing and Condition Monitoring Techniques for Renewable Energy Industrial Assets, M. Papaelias, F.P. García and A. Karyotakis, Ed., Oxford, UK: Butterworth-Heinemann, 2020, capítulo 3, pp. 31-47. [en línea]. Disponible en https://doi.org/10.1016/B978-0-08-101094-5.00003-4 
[13] K. Rothenhagen y F. W. Fuchs, "Doubly Fed Induction Generator Model-Based Sensor Fault Detection and Control Loop Reconfiguration", IEEE Transactions on Industrial Electronics, vol. 56, no. 10, pp. 4229-4238, octubre, 2009. DOI: 10.1109/TIE.2009.2013683.

[14] Z. Liu y L. Zhang, "A review of failure modes, condition monitoring and fault diagnostics methods for large-scale wind turbine bearings", Measurement, vol. 149, enero, 2020. DOI: https://doi. org/10.1016/j.measurement.2019.107002

[15] B. Hahn, et al., "Recommended practices for wind turbine farm data collection and reliability assessment for O\&M optimization", Energy Procedia, vol. 137, octubre, 2017, pp. 358-365. DOI: https://doi.org/10.1016/j.egypro.2017.10.360

[16] A. L. Loría, E. M. Villalobos y C. Piedra, "Modelo de toma de decisiones de mantenimiento basado en la predicción de vida útil para componentes de sistemas eólicos en Costa Rica", Tecnología en Marcha, vol. 30, no. 3, pp. 129-141, octubre, 2017. DOI: https://doi.org/10.18845/tm.v30i3.3279

[17] J. Helsen, C. Peeters, P. Doro, E. Ververs y P.J. Jordaens, "Wind farm operation and maintenance optimization using big data", presentado en 2017 IEEE Third International Conference on Big Data Computing Service and Applications (BigDataService), San Francisco, Estados Unidos, abril 6-9, 2017, pp. 179-184, DOI: 10.1109/BigDataService.2017.27

[18] A. Y. Aguilar Romero y L. F. Tandazo Flores. "Análisis de modos de falla, efectos y criticidad del sistema de inyección de un motor de encendido provocado corsa evolution $1.4 \mathrm{~L}$ empleando herramientas de aprendizaje y clasificación para la programación de mantenimiento", Tesis de Bachillerato, Universidad Politécnica Salesiana, Cuenca, Ecuador, 2017.

[19] ICE. “Tarifa T-CB Ventas a ICE distribución y CNFL S.A”, Instituto Costarricense de Electricidad, San José, Costa Rica, p. 8, 2020. 